\title{
EXPANDING THE SOCLE OF A CODIMENSION 3 COMPLETE INTERSECTION
}

\author{
JESSICA ANN FAUCETT
}

\begin{abstract}
A quotient ring of a regular local ring of embedding dimension 3 has a minimal free resolution that carries a differential graded algebra structure. This structure has been used to classify such rings. For some classes very few examples have been observed. One such class is called B, named for Anne Brown, who discovered and gave the first constructions of rings in this class. Her constructions were the only known rings in that class until recently. In this paper, we show how to obtain Artinian rings in the class B by expanding the socle of a complete intersection.
\end{abstract}

1. Introduction. In this paper, let $Q$ be a regular local ring of embedding dimension 3 with maximal ideal $\mathfrak{m}$ and residue field $k=$ $Q / \mathfrak{m}$. For $I$, an ideal in $Q$, set $R=Q / I$. By Hilbert's syzygy theorem, we know that $R$ has a free resolution over $Q$ of length at most 3. Here, we only consider rings, $R$, with minimal free resolutions of length 3 , i.e., ideals, $I$, of grade 3 . So a minimal free resolution of $R$ over $Q$ takes on the form

$$
F: 0 \longrightarrow F_{3} \stackrel{f_{3}}{\longrightarrow} F_{2} \stackrel{f_{2}}{\longrightarrow} F_{1} \stackrel{f_{1}}{\longrightarrow} F_{0} \stackrel{f_{0}}{\longrightarrow} R \longrightarrow 0 .
$$

We know that $\operatorname{rank}_{Q}\left(F_{0}\right)=1$. Setting $m$ to be the minimal number of generators for $I$, this gives $\operatorname{rank}_{Q}\left(F_{1}\right)=m$. Set $n=\operatorname{rank}_{Q}\left(F_{3}\right)$, and recall that this number is the type of $R$. We know that the Euler characteristic of the deleted minimal free resolution must be 0 . This gives $\operatorname{rank}_{Q}\left(F_{2}\right)=n+m-1$. So a deleted minimal free resolution of $R$ over $Q$ is of the form

$$
F: 0 \longrightarrow Q^{n} \stackrel{f_{3}}{\longrightarrow} Q^{n+m-1} \stackrel{f_{2}}{\longrightarrow} Q^{m} \stackrel{f_{1}}{\longrightarrow} Q \longrightarrow 0 .
$$

In [5], Buchsbaum and Eisenbud proved that $F$ carries the structure of an associative, graded-commutative, differential graded algebra.

2010 AMS Mathematics subject classification. Primary 13C99, 13 H10.

Keywords and phrases. Complete intersection ring, Koszul homology.

Received by the editors on December 19, 2014. 
Graded-commutative means that, for homogeneous elements $a$ and $b$, we have

$$
a b=(-1)^{|a||b|} b a \text { and } a^{2}=0
$$

if $|a|$ is even. Differential graded means that the differential, $f$, satisfies the Leibniz rule:

$$
f(a b)=f(a) b+(-1)^{|a|} a f(b) .
$$

Since $F$ is minimal, $F \otimes_{Q} k=\mathrm{H}\left(F \otimes_{Q} k\right)=\operatorname{Tor}^{Q}(R, k)$ where the second equality is the definition of Tor. The differential graded algebra structure on $F$ is not, in general, unique. However, the induced structure on the graded-commutative algebra $A=\operatorname{Tor}^{Q}(R, k)$ is unique up to isomorphism, and the possible isomorphism classes were identified in two different ways: first, by Weyman [9] in characteristic 0 , and then by Avramov, Kustin and Miller [3] in general.

The isomorphism classes are described explicitly using multiplication tables; however, for our purposes, it is enough to look at the following invariants

$$
p=\operatorname{rank}_{k}\left(A_{1} \cdot A_{1}\right), \quad q=\operatorname{rank}_{k}\left(A_{1} \cdot A_{2}\right), \quad r=\operatorname{rank}_{k} \delta,
$$

where $\delta: A_{2} \rightarrow \operatorname{Hom}_{k}\left(A_{1}, A_{3}\right)$ is defined by $\delta(x)(y)=x y$ for $x \in A_{2}$ and $y \in A_{1}$. The possible classes, along with their corresponding values of these invariants, are given in Table 1 .

TABLE 1.

\begin{tabular}{l||c|c|c}
\hline Class & $p$ & $q$ & $r$ \\
\hline \hline $\mathbf{B}$ & 1 & 1 & 2 \\
$\mathbf{C}(3)$ & 3 & 1 & 3 \\
$\mathbf{G}(r)$ & 0 & 1 & $r \geq 2$ \\
$\mathbf{H}(p, q)$ & $p \geq 0$ & $q \geq 0$ & $r=q$ \\
$\mathbf{T}$ & 3 & 0 & 0 \\
\hline
\end{tabular}

We will be using these invariants along with the values of $m$ and $n$ from above to determine to which class a ring belongs.

For a given ring, $R$, the invariants $p, q$ and $r$ are bounded by $m$ and $n$, see [2], that is, for fixed values of $m$ and $n$, there are finitely possible structures. This leads to the question: which of the classes can actually be realized? For instance, consider class B. When Weyman 
identified the isomorphism classes, he overlooked the class now called B. The class was realized by Brown [4] and thus received its name. In [4], she actually gave constructions for rings in classes $\mathbf{B}$ and $\mathbf{H}(1,2)$ for $n=2$ and $m \geq 4$. In [4], Brown also proved the following.

Fact. If $I$ is a grade 3 perfect ideal and $R=Q / I$ has $m \geq 4, n=2$ and $p>0$, then $R$ is in one of the classes:

(a) $\boldsymbol{B}$ with odd $m$.

(b) $\boldsymbol{H}(1,2)$ with even $m$.

This was all that was known about rings in class $\mathbf{B}$ until recently. In 2014, Painter gave a construction for rings in class $\mathbf{B}$ with $m \geq 5$ and $n=m-3$, see [8]. Painter also gave a specific example of a ring in class $\mathbf{B}$ with $m=6$ and $n=4$. In 2012, Christensen and Veliche found four sporadic examples of rings in class B different from Painter's and Brown's, see [6]. Here is the main result of this paper which is a construction of rings in class $\mathbf{B}$ from complete intersections.

Theorem. Let $Q$ be a regular local ring of embedding dimension 3 with maximal ideal $\mathfrak{m}$ and residue field $k=Q / \mathfrak{m}$. Let $g_{1}, g_{2}, g_{3} \in \mathfrak{m}^{2}$ be a regular sequence in $Q$. For $I=\left(g_{1}, g_{2}\right)+\mathfrak{m} g_{3}$, the quotient $Q / I$ is in class $\boldsymbol{B}$.

2. Notation and example. Now we will offer more insight into the classification by looking deeper at the multiplicative structure of Tor. This will be illustrated with an example where we classify a ring by looking at the multiplication tables.

Let $I$ be an ideal of $Q$, and set $R=Q / I$. The above classification of $R$ is based on the multiplicative structure induced on the gradedcommutative algebra $A=\operatorname{Tor}^{Q}(R, k)$ that comes from the differential graded algebra structure of a minimal free resolution $F$ of $R$ over $Q$. Denote the Koszul complex on the maximal ideal of $Q$ by $K^{Q}$. Recall that $K^{Q}$ is a minimal free resolution of $k$ over $Q$.

We can compute $A=\operatorname{Tor}^{Q}(R, k)$ in two different ways, either by resolving $R$ over $Q$ or by resolving $k$ over $Q$, that is,

$$
\mathrm{H}\left(F \otimes_{Q} k\right)=\operatorname{Tor}^{Q}(R, k)=\mathrm{H}\left(R \otimes_{Q} K^{Q}\right) .
$$


The classification is based on the induced multiplicative structure on $\mathrm{H}\left(F \otimes_{Q} k\right)$ from $F$; however, Avramov proved [1] that this structure is isomorphic to the induced multiplicative structure on $\mathrm{H}\left(R \otimes_{Q} K^{Q}\right)$ from $K^{Q}$. Finding the multiplicative structure on a minimal free resolution $F$ can be difficult; however, we understand $K^{Q}$ and its multiplicative structure because it is an exterior algebra.

Let $x, y, z$ be a set of minimal generators of the maximal ideal $\mathfrak{m}$ of $Q$. Recall that the Koszul complex of $\mathfrak{m}$ over $Q$ takes on the form:

$$
K^{Q}: 0 \longrightarrow Q \stackrel{\partial_{3}=\left[\begin{array}{c}
z \\
-y \\
x
\end{array}\right]}{\longrightarrow} Q^{3} \stackrel{\partial_{2}=\left[\begin{array}{ccc}
-y & -z & 0 \\
x & 0 & -z \\
0 & x & y
\end{array}\right]}{\longrightarrow} Q^{3} \stackrel{\partial_{1}=\left[\begin{array}{lll}
x & y & z
\end{array}\right]}{\longrightarrow} Q \longrightarrow 0 .
$$

Denote a basis for $Q^{3}$ in the first degree by $e_{x}, e_{y}$ and $e_{z}$, where we have

$$
\partial_{1}\left(e_{x}\right)=x, \quad \partial_{1}\left(e_{y}\right)=y, \quad \partial_{1}\left(e_{z}\right)=z .
$$

Then the basis used for $Q^{3}$ in the second degree is $e_{x} \wedge e_{y}, e_{x} \wedge e_{z}$ and $e_{y} \wedge e_{z}$, where we have

$$
\begin{gathered}
\partial_{2}\left(e_{x} \wedge e_{y}\right)=-y e_{x}+x e_{y}, \quad \partial_{2}\left(e_{x} \wedge e_{z}\right)=-z e_{x}+x e_{z}, \\
\partial_{2}\left(e_{y} \wedge e_{z}\right)=-z e_{y}+y e_{z} .
\end{gathered}
$$

Finally the basis used for $Q$ in the third degree is $e_{x} \wedge e_{y} \wedge e_{z}$, where we have

$$
\partial_{3}\left(e_{x} \wedge e_{y} \wedge e_{z}\right)=z\left(e_{x} \wedge e_{y}\right)-y\left(e_{x} \wedge e_{z}\right)+x\left(e_{y} \wedge e_{z}\right) .
$$

The following example illustrates the Theorem. The classification is determined by using the multiplicative structure as induced by the Koszul complex.

Example. Let $k$ be a field, and set $Q=k \llbracket x, y, z \rrbracket$. Consider the $Q$ regular sequence $x^{2}, y^{2}, z^{2}$. Set

$$
I=\left(x^{2}, y^{2}\right)+\mathfrak{m} z^{2}=\left(x^{2}, y^{2}, x z^{2}, y z^{2}, z^{3}\right) .
$$

Three of the generators of $I$ are pure powers of $x, y$ and $z$, so $I$ contains a power of the maximal ideal and $R=Q / I$ is hence Artinian. This gives that $I$ is a grade 3 perfect ideal. We have that $I$ is minimally generated by five elements, so for $R$, we have $m=5$. 
For $d \geq 0$, denote by $R_{d}$ the subspace of $R$ of homogeneous polynomials of degree $d$. The bases for the subspaces are listed in Table 2 .

\section{TABLE 2.}

\begin{tabular}{c|c} 
Subspace & Basis \\
\hline$R_{0}$ & 1 \\
$R_{1}$ & $x, y, z$ \\
$R_{2}$ & $x y, x z, y z, z^{2}$ \\
$R_{3}$ & $x y z$ \\
$R_{4}$ & 0
\end{tabular}

Notice that $z^{2}$ and $x y z$ span the socle of $R$; thus, the type of $R$ is 2 .

Thus, $R$ is a ring with $m=5$ and $n=2$. By Brown's work, we know that $R$ is in the class B if $p>0$. et $A=\mathrm{H}\left(R \otimes_{Q} K^{Q}\right)$. To find a $k$-basis for $A_{1}$ we need five elements that are in the kernel of $\partial_{1}$ and linearly independent over $k$ modulo the image of $\partial_{2}$. The first condition is easily satisfied if we take $x e_{x}, y e_{y}, x z e_{z}, y z e_{z}$ and $z^{2} e_{z}$. For the other condition, view these generators along with the generators of the image of $\partial_{2}$ to see that they are linearly independent over $k$ modulo the image of $\partial_{2}$.

\begin{tabular}{ccccc|ccc} 
& \multicolumn{1}{|c|}{} & $\operatorname{im}_{2}$ & & \\
\hline$x$ & 0 & 0 & 0 & 0 & $-y$ & $-z$ & 0 \\
0 & $y$ & 0 & 0 & 0 & $x$ & 0 & $-z$ \\
0 & 0 & $x z$ & $y z$ & $z^{2}$ & 0 & $x$ & $y$
\end{tabular}

Now consider the multiplication table for $A_{1} \cdot A_{1}$.

\begin{tabular}{c|ccccc}
$A_{1} \cdot A_{1}$ & $x e_{x}$ & $y e_{y}$ & $x z e_{z}$ & $y z e_{z}$ & $z^{2} e_{z}$ \\
\hline$x e_{x}$ & 0 & $x y\left(e_{x} \wedge e_{y}\right)$ & 0 & $x y z\left(e_{x} \wedge e_{z}\right)$ & 0 \\
$y e_{y}$ & & 0 & $x y z\left(e_{y} \wedge e_{z}\right)$ & 0 & 0 \\
$x z e_{z}$ & & & 0 & 0 & 0 \\
$y z e_{z}$ & & & & 0 & 0 \\
$z^{2} e_{z}$ & & & & & 0
\end{tabular}

Recall that the diagonal is zero from the graded-commutative property and the table will be anti-symmetric, so we only need to look at entries above the diagonal. Notice that three nonzero products exist; however, they must be nonzero modulo the image of $\partial_{3}$. To check this, look at 
the three products along with the image of $\partial_{3}$.

\begin{tabular}{ccc|c} 
& & & $\mathrm{im} \partial_{3}$ \\
\hline$x y$ & 0 & 0 & $z$ \\
0 & $x y z$ & 0 & $-y$ \\
0 & 0 & $x y z$ & $x$
\end{tabular}

Clearly, $x y\left(e_{x} \wedge e_{y}\right)$ is not in $\operatorname{im}\left(\partial_{3}\right)$. Thus, $p>0$ and the quotient $Q / I$ is in class B. Note that the other two products are

$$
\partial_{3}\left(-x z\left(e_{x} \wedge e_{y} \wedge e_{z}\right)\right)=x y z\left(e_{x} \wedge e_{z}\right)
$$

and

$$
\partial_{3}\left(y z\left(e_{x} \wedge e_{y} \wedge e_{z}\right)\right)=x y z\left(e_{y} \wedge e_{z}\right),
$$

so we can see that $p=1$ is as it should be for a ring in class $\mathbf{B}$.

Note that it is required for $Q$ be a regular local ring of embedding dimension 3. For example, if $k$ is a field and $Q=k \llbracket x, y, z, w \rrbracket$, then $x^{2}$, $y^{2}, z^{2}$ is still a $Q$-regular sequence; however, if $I=\left(x^{2}, y^{2}\right)+\mathfrak{m} z^{2}$ and $R=Q / I$, then $R$ is a ring whose minimal free resolution is of length 4 . In the above example, the approach used to prove that $R=Q / I$ is in class $\mathbf{B}$ was to look directly at the multiplication structure as induced by the Koszul complex. For the proof of the theorem we will approach the classification from a different perspective which was inspired by work in [7], where a similar technique was used to find non-Gorenstein rings in the class $\mathbf{G}(r)$ from Gorenstein rings.

3. Proof of Theorem. Set $J=\left(g_{1}, g_{2}, g_{3}\right), S=Q / J$ and $R=Q / I$. We have that $J$ is generated by a regular sequence, so $S$ is a complete intersection and must be Artinian. Thus, there exists some natural number $s$ such that $\mathfrak{m}^{s+1} \subseteq J$ while $\mathfrak{m}^{s} \nsubseteq J$. We also have that $\mathfrak{m} J \subseteq I$, so $\mathfrak{m}^{s+2} \subseteq I$, and hence, $R$ is Artinian. This means that $I$ is a grade 3 perfect ideal.

Setting $C=\mathrm{H}\left(S \otimes_{Q} K^{Q}\right)$, since $J$ is generated by a regular sequence, we have $S$ is a complete intersection and is in class $\mathbf{C}(3)$, see [2]. Thus, $C$ is an exterior algebra on $k^{3}$ so the ranks of the homology modules are the following:

$\operatorname{rank}_{k} C_{3}=1, \quad \operatorname{rank}_{k} C_{2}=3, \quad \operatorname{rank}_{k} C_{1}=3, \quad \operatorname{rank}_{k} C_{0}=1$. 
Since $I \subseteq J \subseteq Q$, we have the short exact sequence,

$$
0 \longrightarrow J / I \longrightarrow Q / I \longrightarrow Q / J \longrightarrow 0
$$

which is isomorphic to,

$$
0 \longrightarrow k \longrightarrow R \longrightarrow S \longrightarrow 0 \text {. }
$$

Tensor over $Q$ with the Koszul complex $K^{Q}$ to obtain an exact sequence of $Q$-complexes:

$$
0 \longrightarrow k \otimes_{Q} K^{Q} \stackrel{\alpha}{\rightarrow} R \otimes_{Q} K^{Q} \stackrel{\beta}{\rightarrow} S \otimes_{Q} K^{Q} \longrightarrow 0 .
$$

Set $A=\mathrm{H}\left(R \otimes_{Q} K^{Q}\right)$. This results in an exact sequence of homology modules

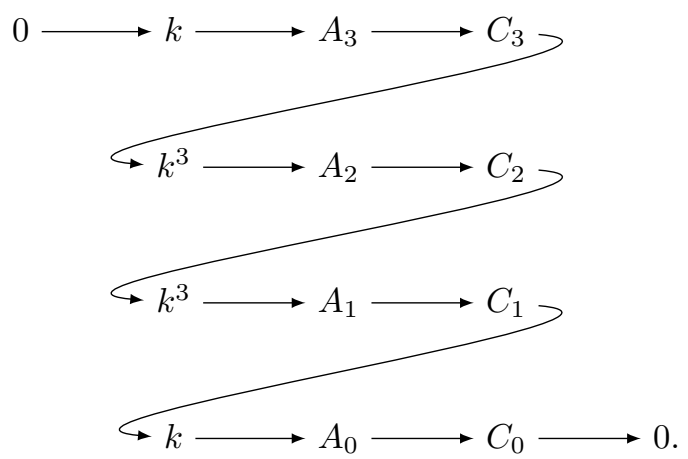

Recall that $\operatorname{rank}_{k} A_{0}=1$. Now we want to calculate $\operatorname{rank}_{k} A_{1}$, which is the same as calculating the number of minimal generators of $I$. Let $x, y, z$ be a set of minimal generators of $\mathfrak{m}$. We know that $I=\left(g_{1}, g_{2}, x g_{3}, y g_{3}, z g_{3}\right)$, so we must show $g_{1}, g_{2}, x g_{3}, y g_{3}$ and $z g_{3}$ are linearly independent modulo $\mathfrak{m} I$. For $\alpha_{i}^{*} \in Q$, suppose

$$
\alpha_{1} g_{1}+\alpha_{2} g_{2}+\alpha_{3}^{x} x g_{3}+\alpha_{3}^{y} y g_{3}+\alpha_{3}^{z} z g_{3} \in \mathfrak{m} I \text {. }
$$

We must show that all $\alpha_{i}^{*} \in \mathfrak{m}$. Since $g_{1}, g_{2}, g_{3}$ is a minimal generating set for $J$ we know that $\alpha_{1}, \alpha_{2}, \alpha_{3}^{x} x+\alpha_{3}^{y} y+\alpha_{3}^{z} z \in \mathfrak{m}$. We also have:

$$
\begin{aligned}
\alpha_{1} g_{1}+\alpha_{2} g_{2}+\alpha_{3}^{x} x g_{3} & +\alpha_{3}^{y} y g_{3}+\alpha_{3}^{z} z g_{3} \\
& =m_{1} g_{1}+m_{2} g_{2}+m_{3}^{x} x g_{3}+m_{3}^{y} y g_{3}+m_{3}^{z} z g_{3},
\end{aligned}
$$


where $m_{i}^{*} \in \mathfrak{m}$. This is equivalent to

$$
\begin{aligned}
\left(\alpha_{1}-m_{1}\right) g_{1}+\left(\alpha_{2}-m_{2}\right) g_{2}+ & \left(\alpha_{3}^{x}-m_{3}^{x}\right) x g_{3} \\
& +\left(\alpha_{3}^{y}-m_{3}^{y}\right) y g_{3}+\left(\alpha_{3}^{z}-m_{3}^{z}\right) z g_{3}=0 .
\end{aligned}
$$

Hence, we have

$$
\left(\left(\alpha_{3}^{x}-m_{3}^{y}\right) x+\left(\alpha_{3}^{y}-m_{3}^{y}\right) y+\left(\alpha_{3}^{z}-m_{3}^{z}\right) z\right) g_{3} \in\left(g_{1}, g_{2}\right) .
$$

Since $g_{1}, g_{2}, g_{3}$ is a regular sequence we must have

$$
\left(\alpha_{3}^{x}-m_{3}^{x}\right) x+\left(\alpha_{3}^{y}-m_{3}^{y}\right) y+\left(\alpha_{3}^{z}-m_{3}^{z}\right) z \in\left(g_{1}, g_{2}\right) \subseteq \mathfrak{m}^{2} .
$$

Since $x, y, z$ are linearly independent modulo $\mathfrak{m}^{2}$ the coefficients $\alpha_{3}^{*}-$ $m_{3}^{*} \in \mathfrak{m}$. Since all $m_{3}^{*} \in \mathfrak{m}$, we must have all $\alpha_{3}^{*} \in \mathfrak{m}$. Hence, $g_{1}, g_{2}, x g_{3}, y g_{3}, z g_{3}$ is a minimal generating set for $I$, and we have $\operatorname{rank}_{k} A_{1}=5$.

The rank nullity theorem allows us to calculate the ranks of the maps in (3.2) up to $A_{2}$. Recall $\alpha$ and $\beta$ from equation (3.1). Set $\phi=$ $\mathrm{H}(\beta): A \rightarrow C$. This gives the following exact sequence where the numbers below the arrows denote the ranks of the map:

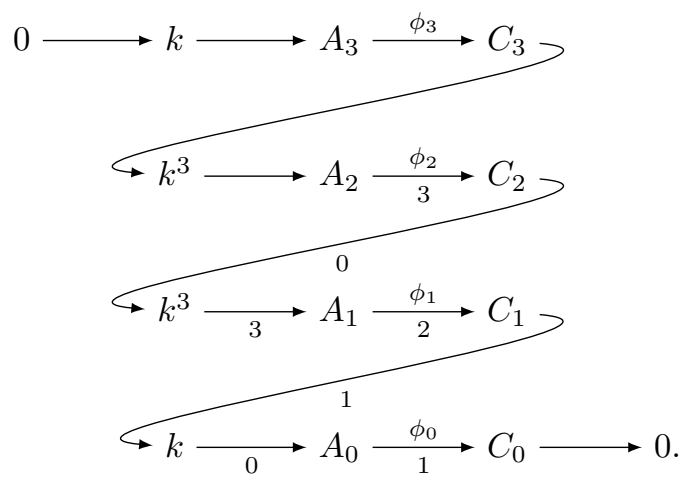

Since the map inducing $\beta$ is a map of $Q$-algebras, $\beta$ is a map of differential graded $Q$-algebras. On the other hand, $\alpha$ is not due to the map inducing since it is only a map of $Q$-modules. Thus, $\phi$ is a map of graded-commutative $k$-algebras. This gives that $\phi$ respects the multiplicative structure on $A$ and $C$. 
Since $C$ is the exterior algebra on $k^{3}$, there is a basis $c_{1}, c_{2}, c_{3}$, for $C_{1}$ such that $c_{1} c_{2}, c_{1} c_{3}, c_{2} c_{3}$ is a basis for $C_{2}$. We have the rank of the map from $A_{1}$ to $C_{1}$ is 2 so, without loss of generality, there exists $0 \neq a_{1}, a_{2} \in A_{1}$ such that

$$
\phi_{1}\left(a_{1}\right)=c_{1}, \quad \phi_{1}\left(a_{2}\right)=c_{2} .
$$

Since $\phi$ respects the multiplicative structure, we must have $\phi_{2}\left(a_{1} a_{2}\right)=$ $c_{1} c_{2}$, and hence, $0 \neq a_{1} a_{2} \in A_{2}$. Thus, for $R$, we have $p>0$.

It follows that we have $R$ is a ring with $m=5$ and $p>0$. This means that, by Brown's theorem, if $n=2$, then $R$ is a ring in the class B.

According to equation (3.2) we have:

$$
1=\operatorname{rank}_{k} k \leq \operatorname{rank}_{k} A_{3} \leq \operatorname{rank}_{k} k+\operatorname{rank}_{k} C_{3}=2 .
$$

So $n=\operatorname{rank}_{k} A_{3}$ is either 1 or 2. It is shown [5] that, in the case where $n=1$ and $m$ is odd with $m \geq 5$, the ring is Gorenstein with $r=m$. Thus, if $n=1$, then $R$ is in $\mathbf{G}(5)$. Recall that rings in the class $\mathbf{G}(r)$ have $p=0$, which is a contradiction. Thus, $n=2$, and the proof is complete.

Acknowledgments. I would like to thank my advisor Lars W. Christensen for his guidance and insight on this topic and in the writing of this paper.

\section{REFERENCES}

1. Luchezar L. Avramov, Small homomorphisms of local rings, J. Algebra 50 (1978), 400-453.

2. , A cohomological study of local rings of embedding codepth 3, J. Pure Appl. Algebra 216 (2012), 2489-2506.

3. Luchezar L. Avramov, Andrew R. Kustin and Matthew Miller, Poincaré series of modules over local rings of small embedding codepth or small linking number, J. Algebra 118 (1988), 162-204.

4. Anne E. Brown, A structure theorem for a class of grade three perfect ideals, J. Algebra 105 (1987), 308-327.

5. David A. Buchsbaum and David Eisenbud, Algebra structures for finite free resolutions, and some structure theorems for ideals of codimension 3, Amer. J. Math. 99 (1977), 447-485.

6. Lars Winther Christensen and Oana Veliche, Local rings of embedding codepth 3: Examples, Alg. Rep. Theor. 17 (2014), 121-135. 
7. Lars Winther Christensen, Oana Veliche and Jerzy Weyman, Trimming a Gorenstein ideal, arXiv: 1512.02720.

8. Jared L. Painter, Planar graphs and the Koszul algebra structure for trivariate monomial ideals, in Connections between algebra, combinatorics, and geometry, Springer Proc. Math. Stat. 76, Springer, New York, 2014.

9. Jerzy Weyman, On the structure of free resolutions of length 3, J. Algebra 126 (1989), 1-33.

Western Governors University, 4001 S. 700 E Suite 700, Salt Lake City, UT 84107

Email address: jessica.faucett@wgu.edu 